\title{
A Decision Support System For Audit Staff Scheduling Of Multiple And Large-Scaled Engagements
}

\author{
C. Janie Chang, (E-mail: chang_j@cob.sjsu.edu), San Jose State University
}

\begin{abstract}
As raising cost of audit and shrinking audit market, the management of accounting firms have put into more efforts on increasing productivity by improving audit planning, upgrade audit technology and cost control. One avenue to accomplish cost savings while maintaining high audit quality is to refine staff scheduling. This study proposes a different approach to resolving audit staff scheduling for multiple and large engagements. The major advantage of the proposed method is to solve staff resource allocation problems by alleviating the limitations of both linear and integer programming. In addition, the objectives and constraints of models proposed in this study can be modified easily to adjust for firm-specific situations.
\end{abstract}

\section{Introduction}

D uring the past two decades, the public accounting profession has encountered many new challenges. For example, due to rapid globalization of the world economy, many accounting firms, such as the Big 5, have had to restructure their operations and to expand their business activities overseas in order to provide better services to their clients. Moreover, due to competitive bidding practices, CPA firms must explore different approaches to providing high quality service at the lowest costs. One possible avenue to control audit cost is to schedule its professional staff efficiently.

The objective of implementing systematic scheduling approaches is to minimize audit costs by fully utilizing available audit resources (i.e., reduce audit staff's idle time). Rely on scheduling manager's personal experience to find the optimal solution for scheduling may be feasible when the size of a firm is relatively small. However, when the firm size increases, it is very difficult, if not impossible, for a manager to rely simply on his/her previous experience to schedule the audit professionals' work efficiently. Sub-optimal audit staff scheduling may end up causing large-sized firms to suffer substantial losses. To avoid the costs of inefficient scheduling, accounting firms need to implement a systematic approach to assigning their audit staff. As James F. Harrington, a national consulting and SEC technical services partner at PricewaterhouseCoopers, indicated in his firm's 1999 academic workshop, although there are several computer software available in arranging staff assignments, the audit-staff scheduling continues to be one of the important issues for major accounting firms, particularly for the attestation and consulting services. An effective decision support system for staff scheduling can definitely reduce potential loss of human resource, thus increase an accounting firm's profitability.

The purpose of this study is to propose a systematic scheduling approach by adopting a decision support system (DSS). The system established in this study will assist CPA firms in efficiently scheduling their audit staff for large-scaled operations in a multiple audit engagement environment. To fulfill scheduling objectives, this study explicitly considers engagement deadlines, the precedent relationship among audit tasks, and the efficiency variance among auditors with different levels of expertise.

Readers with comments or questions are encouraged to contact the authors via email. 
The remainder of this paper is organized as follows. Next section provides the literature background of audit scheduling related issues. The second section states and formulates the scheduling problem. The third section describes a heuristic algorithm and constructs a hypothetical case to illustrate the proposed algorithm. In the fourth section, a pair-wise exchange algorithm is presented to demonstrate how it would systematically improve the scheduling results from the heuristic model. Concluding remarks and future research directions are highlighted in the last section.

\section{Background}

Prior research in audit staff scheduling has attempted to solve scheduling problems using linear programming, integer programming, or goal programming. The objectives of the approaches these studies proposed were to optimize the auditors' assignments by meeting one or more objective functions with different constraints ${ }^{\mathrm{i}}$. For example, Summers (1972) presented a linear programming model that considers the actual number of hours an auditor needs to complete a task as well as the time limitations imposed by the engagement deadline. Balachandran and Zoltners (1981) developed an integer programming model (BZ model) to solve audit-staff scheduling problems. The BZ model eliminates some limitations of the linear programming approach in assigning auditors; for example, concurrent assignment of audit tasks to an auditor and determination of an assignment's practicability are no longer problematic. Furthermore, Balachandran and Steuer (1982) apply a multiple-objective linear programming approach and propose an interactive model to derive the weighting factors for audit planning. Instead of assigning weighting factors at the beginning, they allow the decision-makers to assign the weights of each objective through the interactive selection of their most preferred alternatives. After a few iterations, the final weighting factors may be derived. Balachandran and Steuer argue that the weighting factors derived in this manner are much more representative than those pre-specified for multiple objectives. Overall, these prior studies have provided a reasonable framework for audit-staff scheduling. However, these studies did not adequately address issues related to the sequence of audit tasks or the duration of those tasks.

One important characteristic of a typical audit engagement is the sequence of sets of interrelated tasks, such as internal control assessment and substantive tests. To efficiently schedule audit staff, it is imperative for a scheduling manager to consider the sequence of these tasks. Chan and Dodin (1986) extend the BZ model by considering important and practical constraints, such as precedence relationships, arrival times of audit tasks and engagement due dates. They construct a PERT network with explicit consideration of the precedence relationships and arrival times for multiple concurrent engagements. Furthermore, Dodin and Chan (1991) further expand their 1986 study by utilizing earliest start time and latest completion time in the activity network to enhance the linear integer program's efficiency. To test the practical applicability of the proposed scheduling approach, Dodin and Chan apply the model to a real life audit scheduling case, and the model does generate an optimal schedule for the firm.

Following the same research line, Gardener, Heufner, and Lotfi (1990) modify the BZ model by considering busy season phenomena. Factors such as reducing overtime and maintaining scheduling flexibility by allowing staff-level changes and schedule rearrangement were explicitly considered in their multi-period audit staff planning model. Similarly, Hwang (1998) proposes a heuristic approach to audit scheduling that balances workload and reduces unnecessary overtime. In this study, Hwang claims the proposed scheduling approach would provide auditors a higher level of job satisfaction by reducing unnecessary overtime. While the PERT-type activity network scheduling approach has been developed and studied intensively in both manufacturing and service sectors including accounting firms (Chan and Dodin 1986; Dean, Denzler, and Watkins 1992; Dodin and Chan 1991; Hamburger 1987; Krogstad et al. 1977; Thompson 1996; Ying 1996), optimal solutions to audit scheduling problems in multiple and large engagement settings have yet to be developed.

To accomplish this objective, I first construct a hypothetical case to demonstrate how the proposed model can be adapted to solve scheduling problems heuristically. Then, I illustrate how to systematically improve the model's efficiency by alleviating the limitations on the number of variables and constraints, and the degree of computational complexity that arise when either linear or integer programming are used. Finally, I present how the improved model provides an optimal solution for audit staff scheduling problems in multiple and large audit engagements. 


\section{Problem Statement and Formulation}

In general, audit staff scheduling can be structured to meet a CPA firm's operational objectives. As in other scheduling approaches, a particular scheduling objective function (or functions) that is subject to a number of constraints can be formulated into this proposed approach. Depending upon the practical requirements, a scheduling manager can specify the objective functions with factors such as reducing the engagement (or task) duration and/or eliminating lateness penalties, as well as prioritizing them.

In this study, the constraints of the objective functions include the following: (1) different levels of auditor efficiency, ${ }^{\text {ii }}$ (2) precedence relationships among audit tasks, (3) due dates of engagements, (4) auditors' expertise with respect to different audit tasks, ${ }^{\text {iii }}$ (5) each audit task is to be completed by one auditor, and (6) an auditor's inability to process concurrent tasks simultaneously. ${ }^{\text {iv }}$ The overall objective function of this scheduling approach is to minimize the engagement completion time, so the audit cost for a given engagement can be minimized.

The activity network is used to represent the interrelationships among audit tasks and to structure the entire audit engagement (Chan and Dodin 1986; Dodin and Chan 1991; Hwang 1998). The network usually consists of the duration time and the various constraints of each task, such as earliest start time and latest completion time. In practice, this activity network can also be used to organize an audit team and assign audit tasks to staff. Precedence constraints are considered in the network since the connections of a task to related tasks can be specified clearly in a single graph. This type of representation will be utilized throughout the paper.

The activity network $\left(G_{t}\right)$ is a directed, acyclic graph, $G_{t}=\left(V_{t}, E_{t}\right)$, where $V_{t}=\{i: i=1,2, \ldots, N\}$ is the set of nodes denoting the tasks of an audit engagement, and $E_{t}=\{\langle i, j\rangle: i, j=1,2, \ldots, N ; i \neq j\}$ is the set of directed edges representing the precedence relationship that task $i$ must be completed before task $j$ can be started. Each node of the activity network is parameterized by a 3 -tuple $\left(s_{i}, d_{i}, a_{i}\right)$, where $s_{i}$ is the service demand of task $i$, measured in terms of a particular measurement unit or report unit such as number of audit hour, while $d_{i}$ is the due date of task $i$, and $a_{i}$ is the set of auditors to whom task $i$ can be assigned. It is also assumed, without loss of generality, that an activity network $G_{t}$ consists of a start task and a terminal task. In other words, in the activity network $G_{t}$, each task can be reached either by moving forward from the start task or by moving backward from the terminal task. If an activity network does not have a start task or a terminal task, a dummy start task/terminal task must be added to the network such that the service unit of the start task is zero. For notational convenience, it is assumed that the terminal task corresponds to task $N$ of the activity network, $G_{t}$.

Similarly, the set of auditors for a specific engagement can be represented as $V_{p}$, where $V_{p}=\{p: p=1,2$, $\ldots, M\}$ and $M$ is the total number of auditors. Auditor $p$ can be parameterized by a 3 -tuple $\left(\mu_{p}, e_{\mathrm{p}}, R_{p}\right.$ ) where $\mu_{p}$ is the efficiency (i.e., service rate or productivity) of auditor $p$, where $e_{p}$ is the salary rate for auditor $p$, and where $R_{p}$ is the total number of time units that auditor $p$ can work on the activity network during the planning horizon. It is assumed that task scheduling is non-preemptive, i.e., once an auditor starts working on a task, he/she cannot start any other task until the assigned task is finished. The required time units for the processing of task $i$ by auditor $p$ can be calculated by the service demand of task $i$ divided by the service rate of auditor $p$ (i.e., $s_{i} / \mu_{p}$ ). The cost for this assignment will be the salary rate of auditor $p$ multiplied by the processing time (i.e., $e_{p}\left(s_{i} / \mu_{p}\right)$ ) while ignoring other expenditures. These two values will be used later in formulating the audit-scheduling problem. Formally, audit staff scheduling is a partition of the task set $V_{t}=\{1,2, \ldots, N\}$ into $M$ ordered sets $T_{1}, T_{2}, \ldots, T_{M}$ :

$T_{p}=\left\{p_{1}, p_{2}, \ldots, p_{k}\right\}, k \geq 1, p_{i}$ is the $i^{\text {th }}$ task assigned to auditor $p$, and

$\cup 1 \leq p \leq M T_{p}=\{1,2, \ldots, \mathrm{N}\}$.

This partition satisfies the precedence constraints of the activity network, tasks due dates, different levels of auditors' expertise, and auditors' available time units during the planning horizon. 
The ordered sets $T_{1}, T_{2}, \ldots, T_{M}$ are disjoint, i.e., no single task is assigned to more than one auditor. Let $\rho_{i}$ denote the set of immediate parent(s) of task $i$ in the activity network, $G_{t}$. Then, minimizing the total completion time can be stated succinctly as follows:

$\operatorname{Min} C_{N}\left(T_{1}, T_{2}, \ldots, T_{M}\right)$,

$\left\{T_{1}, T_{2}, \ldots, T_{M}\right\}$

subject to:

$\sum s_{i} / \mu_{p} \leq R_{p}($ resource constraint for auditor $p)$,

$i \in T_{p}$ and $p \in a_{i}$

$C_{i}\left(T_{1}, T_{2}, \ldots, T_{M}\right) \leq d_{i}$ (due date of task $\left.i\right), 1 \leq i \leq N$,

where $C_{i}\left(T_{1}, T_{2}, \ldots, T_{M}\right)$ is the completion time of task $i$ under the assignment $\left(T_{1}, T_{2}, \ldots, T_{M}\right)$ and $N$ is the terminal task of the activity network. The sequence of assignments must satisfy the precedence constraints; that is, the execution of task $i$ cannot begin until all the parent tasks in $\rho_{i}$ have been completed.

When the objective is to minimize the total audit $\operatorname{cost}^{\mathrm{vi}}$, the objective function in equation (2) can be modified as follows:

Min $\mathrm{E}=\sum \sum e_{p}\left(s_{i} / \mu_{p}\right)$,

$1 \leq p \leq M \quad i \in T_{p}$ and $p \in a_{i}$

subject to the constraints stated in equations (3) and (4).

\section{A Heuristic Model}

Based on the critical path method (CPM), the level of task $i, L_{i}$, is defined as the minimum amount of time required from the starting to execute task $i$ to the overall completion of all of the audit engagements. Without loss of generality, it is assumed that task $l$ is the starting task, task $N$ is the terminal task of the network activity, $\alpha_{i}$ is the set of immediate successors (child tasks) of task $i$, and $\beta_{i}$ is the set of immediate predecessors (parent tasks) of task $i$. The level of task $i, L_{i}$, is given by

$L_{i}=s_{i} / \mu_{f}+\max L_{j}$,

$j \in \alpha I$

where $f$ is the auditor with the highest efficiency (i.e., fastest service rate or highest level of productivity). Note that $L_{1}$ is the lower bound of required time unit for the completion of all of the engagements. This means that no matter how many auditors are allocated to the engagements, the completion time is always greater than or equal to $L_{i}$. From the computed levels, a priority list represented using [1] [2] ... [N] is constructed by sorting $L_{i}$ in a nonincreasing order. If there is a tie between any two tasks, the task with more child tasks will be given a higher priority in the list (i.e., most immediate successors first). Audit tasks are assigned one by one according to the priority list, with the local optimal solution considered first until all the tasks have been assigned. This heuristic is called the Critical Path Method/Most Immediate Successors First (CPM/MISF) (Kasahara and Narita 1984). Assigning tasks according to the non-increasing list would satisfy the precedence constraints.

Proof: Suppose that task $i$ is assigned before task $j$. According to the above equation (6), $L_{i} \geq L_{j}$. Assuming that assigning task $i$ before task $j$ violates the precedence constraints, i.e., if we traverse task $i$ backwards toward the start task, we can find task $j$ along the path. From the definition of level, the level of a parent task must 
be greater than the level of that specific task because the service demand of any task is always greater than zero. Since we find task $j$ when we traverse backwards from task $i$, we know that $L_{j}>L_{i}$ is a contradiction.

The due dates of tasks, available hours of auditors, and auditors' levels of expertise restrict the feasibility of optimum scheduling in assigning each task. The precedence constraints, however, impose additional synchronization delays, since task $i$ cannot begin execution until all its parent tasks have been completed. To derive the scheduling equation, let $A_{i}$ denote the set of auditors to which a task $i$ can be assigned without violating the engagement due date and the total available audit hours, i.e.,

$$
A_{i}=\left\{p: \quad \sum \quad s_{i} / \mu_{p} \leq R_{p}\right\} \quad i \in T_{p} \text { and } p \in a_{i}
$$

The completion time of task $i$ when assigned to auditor $p, p \in A_{i}$, is given by

$$
\begin{gathered}
C_{i}\left(T_{1}, T_{2}, \ldots, T_{p} \cup i, . . T_{M}\right)=\operatorname{Max} \\
p \in A_{i}
\end{gathered}
$$

and the time units to complete task $i$ must be less than the time units needed until the engagement due date, $d_{i}$, i.e.,

$C_{i}\left(T_{1}, T_{2}, \ldots, T_{p} \cup i, . . T_{M}\right) \leq d_{i}$.

\section{The Proposed Heuristic Scheduling Model}

Given a directed task activity network graph $G_{t}=\left(V_{t}, E_{t}\right)$ with parameters $\left(s_{i}, d_{i}, a_{i}\right)$ and auditors with parameters $\left(\mu_{p}, e_{\mathrm{p}}, R_{p}\right)$, the heuristic scheduling model computes the assignments of tasks $\left(T_{1}, T_{2}, . ., T_{p}, . ., T_{M}\right)$, where $T_{p}$ is the ordered set of tasks allocated to auditor $p$.

Step 1: Determine the level of each task.

$\mathrm{S}=\{\mathrm{N}\}$

Repeat until $\mathrm{S}=\phi$

Select a task $i$ of S such that no successors of task $i$ appear in S.

Compute the level of task $i$ according to equation (6).

$\mathrm{S}=\mathrm{S}-\{i\} \cup \beta_{i}$

Step 2: Construct a priority list [1] [2] ... [N] by sorting $L_{i}$ in a non-increasing order.

Break ties on the basis of number of successors.

Step 3: Assign tasks to auditors.

For $\mathrm{j}=1$ to $N \quad$ do

$i=[\mathrm{j}]$

From the feasible set of auditors $A_{i}$ via equation (7)

Find the auditor $p$ with the minimum $C_{i}$ or $e_{p}\left(s_{i} / \mu_{p}\right)$ via

equations (3), (4), (5), (7), (8) and (9)

Break ties on the basis of the minimum utilization of auditors end do

\section{An Illustrative Example}

For clarity purposes, I assume all staff process the same level of professional expertise to perform all audit tasks for the engagement. The maximum work hours for each auditor during the planning horizon is assumed to be 25 , i.e., $R_{1}=R_{2}=R_{3}=25$. The due date of tasks are assumed to be $d_{1}=10, d_{2}=15, d_{3}=15, d_{4}=15, d_{5}=25$, $d_{6}=25, d_{7}=25$, and $d_{8}=30$. In Figure 1, the number inside a node represents a task and the number near a node represents the service demand of that task. The objective function in this example is to minimize the total comple- 
tion time for the engagement.

The levels $L_{1}, L_{2}, \ldots L_{8}$ in Figure 1 can be computed systematically. To calculate the level of each task and the lower bound of completion time for the entire engagement, it is necessary to utilize the highest efficiency rate among all auditors. For instance, it is assumed that the highest efficiency rate among assigned auditors is one per time unit (e.g., hour or day). To calculate the levels, start from the terminal task and trace backwards toward the start task until all the levels are computed (see Step 1 of the proposed heuristic model). Since task 8 is the terminal task, $L_{8}=5 . L_{7}=\mathrm{s}_{7}+L_{8}=13$. Similarly, $L_{6}=12, L_{5}=9, L_{4}=\mathrm{s}_{4}+\operatorname{Max}\left(L_{6}, L_{7}\right)=17, L_{3}=\mathrm{s}_{3}+L_{5}=14, L_{2}$ $=\mathrm{s}_{2}+L_{5}=15$, and $L_{1}=\mathrm{s}_{1}+\operatorname{Max}\left(L_{2}, L_{3}, L_{4}\right)=23$. Sorting the calculated levels according to the nonincreasing order, we get $L_{1}>L_{4}>L_{2}>L_{3}>L_{7}>L_{6}>L_{5}>L_{8}$. Hence, the priority list of the engagement is [1] $=$ $1,[2]=4,[3]=2,[4]=3,[5]=7,[6]=6,[7]=5$, and $[8]=8$.

\section{Figure 1: An illustration of the activity network of an engagement}

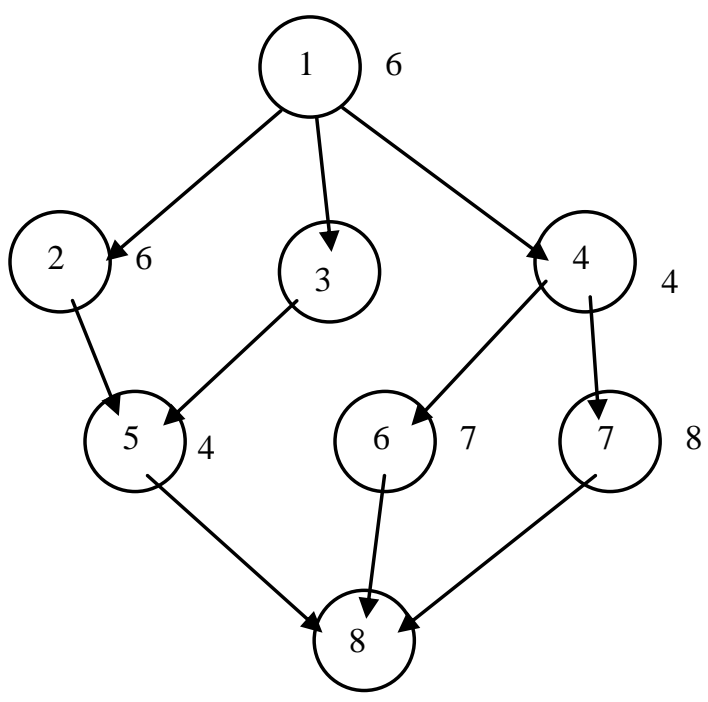

There are several important features implied in this engagement. First, no matter how many auditors are assigned to this engagement, it takes at least 23 time units to complete the all needed audit activities. Second, whenever task $i$ is started, it takes at least $L_{i}$ more time units from the start of task $i$ to the completion of the engagement. In other words, the critical path from the start of task $i$ to completion of the terminal task will take, at least, $L_{i}$ units of time. Finally, one task cannot be started until all its parent tasks have been completed, because of the precedence relationships. For example, the auditor must wait to start task 5 until both tasks 2 and 3 are finished. Once the priority list has been derived, audit tasks can be assigned according to Step 3 in the proposed heuristic model.

To demonstrate how the model works, the following six cases are simulated using the heuristic scheduling approach and the activity networks. ${ }^{\text {vii }}$ Cases 1, 2 and 3 assume the available auditor(s) possess the same level of efficiency and expertise. Case 4 assumes auditors with different levels of efficiency and equal expertise are assigned to the engagement. Case 5 assumes auditors with different levels of efficiency and different levels of expertise are assigned to the engagement. Finally, Case 6 is developed based on the same assumption in Case 5.

\section{Case 1 Only one auditor is assigned to the engagement.}

Assume the efficiency of the auditor assigned to the engagement is one per time unit. Since all the audit tasks will be assigned to just one auditor, the completion time of the engagement is the sum of the service demands of all audit tasks, 45. In this case, the due dates cannot be met because the total available audit time units $\left(R_{1}=25\right)$ are not enough for this engagement. This means more auditors need to be assigned to the engagement if the ac- 
counting firm is to avoid an engagement lateness penalty.

Case 2: $\quad$ Two auditors with equal efficiency and expertise are assigned to the engagement.

To solve the problem of engagement lateness stated in Case 1, let's assume two auditors (1 and 2) are assigned to the engagement and both of them are equally efficient in performing assigned audit tasks at one per time unit (e.g., hour or day). According to the proposed heuristic model, the completion time of the heuristic model is reduced from 45 in Case 1 to 29 (see Table 1 for the simulation results). Although there is a substantial reduction of completion time, the deadline of the engagement still cannot be met under this arrangement.

Table 1 Simulation Results of Case 2

\begin{tabular}{|ccccc|}
\hline Audit Task & Auditor & Start Time & Completion Time & Level \\
1 & 1 & 0.00 & 6.00 & 23.00 \\
4 & 2 & 6.00 & 10.00 & 17.00 \\
2 & 1 & 6.00 & 12.00 & 15.00 \\
3 & 2 & 10.00 & 15.00 & 14.00 \\
7 & 1 & 12.00 & 20.00 & 13.00 \\
6 & 2 & 15.00 & 22.00 & 12.00 \\
5 & 1 & 20.00 & 24.00 & 9.00 \\
8 & 2 & 24.00 & 29.00 & 5.00 \\
\hline
\end{tabular}

Case 3: $\quad$ Three auditors with equal efficiency and expertise are assigned to the engagement.

Assume the third auditor (auditor 3) assigned to the engagement possesses the same level of efficiency and expertise as auditors 1 and 2. Based on the simulation results presented in Table 2, the completion time of the engagement is 23 , which is equivalent to $L_{1}$. Since the completion time of the engagement is equal to the level of critical path on the start task, adding more auditors to this engagement will not reduce the completion time any further. Therefore, in this case, the scheduling manager should assign a total of three auditors to the engagement.

To further demonstrate the proposed model, the same level of expertise among auditors is no longer assumed. Suppose that auditors assigned to the engagement possess different levels of efficiency: auditors 1, 2 and 3 with efficiency rates of 1, 1, and 1.5, respectively. Since the highest efficiency rate among three auditors is auditor 3 at 1.5 , the levels of each audit task assigned to him/her are reduced by one-third of the original level. The heuristic model tends to assign audit tasks to the third auditor because of his/her level of efficiency. The simulation results are presented in Table 3. In this case, the auditors assigned to the engagement are able to complete all the audit tasks before deadline.

Table 2 Simulation Results of Case 3

\begin{tabular}{|ccccc|}
\hline Audit Task & Auditor & Start Time & Completion Time & Level \\
1 & 1 & 0.00 & 6.00 & 23.00 \\
4 & 3 & 6.00 & 10.00 & 17.00 \\
2 & 2 & 6.00 & 12.00 & 15.00 \\
3 & 1 & 6.00 & 11.00 & 14.00 \\
7 & 3 & 10.00 & 18.00 & 13.00 \\
6 & 1 & 11.00 & 18.00 & 12.00 \\
5 & 2 & 12.00 & 16.00 & 9.00 \\
8 & 2 & 18.00 & 23.00 & 5.00 \\
\hline
\end{tabular}


Case 4: $\quad$ Three auditors with different levels of efficiency but the same level of expertise are assigned to the engagement.

Table 3 Simulation Results of Case 4

\begin{tabular}{|ccccc|}
\hline Audit Task & Auditor & Start Time & Completion Time & Level \\
1 & 3 & 0.00 & 4.00 & 15.33 \\
4 & 3 & 4.00 & 6.67 & 11.33 \\
2 & 1 & 4.00 & 10.00 & 10.00 \\
3 & 2 & 4.00 & 9.00 & 9.33 \\
7 & 3 & 6.67 & 12.00 & 8.67 \\
6 & 2 & 9.00 & 16.00 & 8.00 \\
5 & 1 & 10.00 & 14.00 & 6.00 \\
8 & 3 & 16.00 & 19.33 & 3.33 \\
\hline
\end{tabular}

Case 5: $\quad$ Three auditors with different levels of efficiency and different levels of expertise are assigned to the engagement.

Case 5 assumes that three auditors assigned to the engagement not only have different levels of efficiency (i.e., 1, 1, and 1.5) but also possess different levels of expertise. In a real public accounting environment, auditors are assigned to different tasks based on their prior experience and expertise, and each audit engagement would have auditors with different levels of expertise involved. For example, senior auditors in the same CPA firm, in general, should be able to plan an audit, evaluate a client's internal controls, and conduct analytical review procedures. However, some of them may specialize in financing aspects, while others may excel at tax planning for clients involved in merger and acquisition activities. In any audit engagement, it is necessary to have auditors performing different tasks according to their experience and expertise. Therefore, one constraint is added to represent the different levels of expertise among three auditors in Case 5.

Let's assume that auditor 1 can perform tasks 1, 4, 6, 7, and 8; auditor 2 can perform tasks, 2, 3, 4, 5, 6, 7, and 8 ; auditor 3 can perform tasks 3,5 , and 8 . Since auditor 3 has the highest efficiency rate, the levels of each audit task assigned to auditor 3 are reduced to two thirds of the original level. The simulation results of Case 5 are presented in Table 4. According to Table 4, the total completion time for Case 5 is 26.33 , which is over the total time allowed of 25. That is, given three auditors with the efficiency and expertise constraints, the engagement cannot be completed before the deadline. The only way to avoid the lateness penalty is to add more auditor(s) to the engagement.

Table 4 Simulation Results of Case 5

\begin{tabular}{|c|c|c|c|c|}
\hline Audit Task & Auditor & Start Tim & pletion Time & Level \\
\hline 1 & 1 & 0.00 & 6.00 & 15.33 \\
\hline 4 & 2 & 6.00 & 10.00 & 11.33 \\
\hline 2 & 2 & 10.00 & 16.00 & 10.00 \\
\hline 3 & 3 & 6.00 & 9.33 & 9.33 \\
\hline 7 & 1 & 10.00 & 18.00 & 8.67 \\
\hline 6 & 2 & 16.00 & 23.00 & 8.00 \\
\hline 5 & 3 & 16.00 & 18.67 & 6.00 \\
\hline 8 & 3 & 23.00 & 26.33 & 3.33 \\
\hline
\end{tabular}

Case 6: Three auditors with different levels of efficiency and different levels of expertise are assigned to two engagements. 
It is rather common for auditors to be involved in multiple engagements simultaneously. To demonstrate how the proposed heuristic scheduling approach can effectively solve the auditor assignment issue, let's assume auditors with different levels of efficiency and different level of expertise are assigned to two engagements. To handle multiple engagements, dummy nodes need to be added to form the combined activity network so that the starting and ending tasks are clear in the model. To simplify the illustration, two identical engagements are used in Case 6 as shown in Figure 2. A dummy node, 0, and another dummy node, 17, are added to the activity network to combine these two engagements. Node 0 is the starting task and node 17 is the ending task. Both node 0 and node 17 do not require any time from the auditors. To simplify the discussion, all constraints for this case are identical to those of Case 5. The simulation results of Case 6 for two engagements are presented in Table 5.

Table 5 Simulation Results of Case 6

\begin{tabular}{|ccccc|}
\hline Audit Task & Auditor & Start Time & Completion Time & Level \\
0 & 1 & 0.00 & 0.00 & 15.33 \\
9 & 1 & 0.00 & 6.00 & 15.33 \\
1 & 1 & 6.00 & 12.00 & 15.33 \\
4 & 2 & 12.00 & 16.00 & 11.33 \\
12 & 1 & 12.00 & 16.00 & 11.33 \\
10 & 2 & 16.00 & 22.00 & 10.00 \\
2 & 2 & 22.00 & 28.00 & 10.00 \\
3 & 3 & 12.00 & 15.33 & 9.33 \\
11 & 3 & 15.33 & 18.67 & 9.33 \\
15 & 1 & 16.00 & 24.00 & 8.67 \\
7 & 1 & 24.00 & 32.00 & 8.67 \\
6 & 2 & 28.00 & 35.00 & 8.00 \\
14 & 1 & 32.00 & 39.00 & 8.00 \\
5 & 3 & 28.00 & 30.67 & 6.00 \\
13 & 3 & 30.67 & 33.33 & 6.00 \\
8 & 3 & 35.00 & 38.33 & 3.33 \\
16 & 3 & 39.00 & 42.33 & 3.33 \\
17 & 3 & 42.33 & 42.33 & 0.00 \\
\hline
\end{tabular}


Figure 2: An illustration of the activity network of multiple engagements.

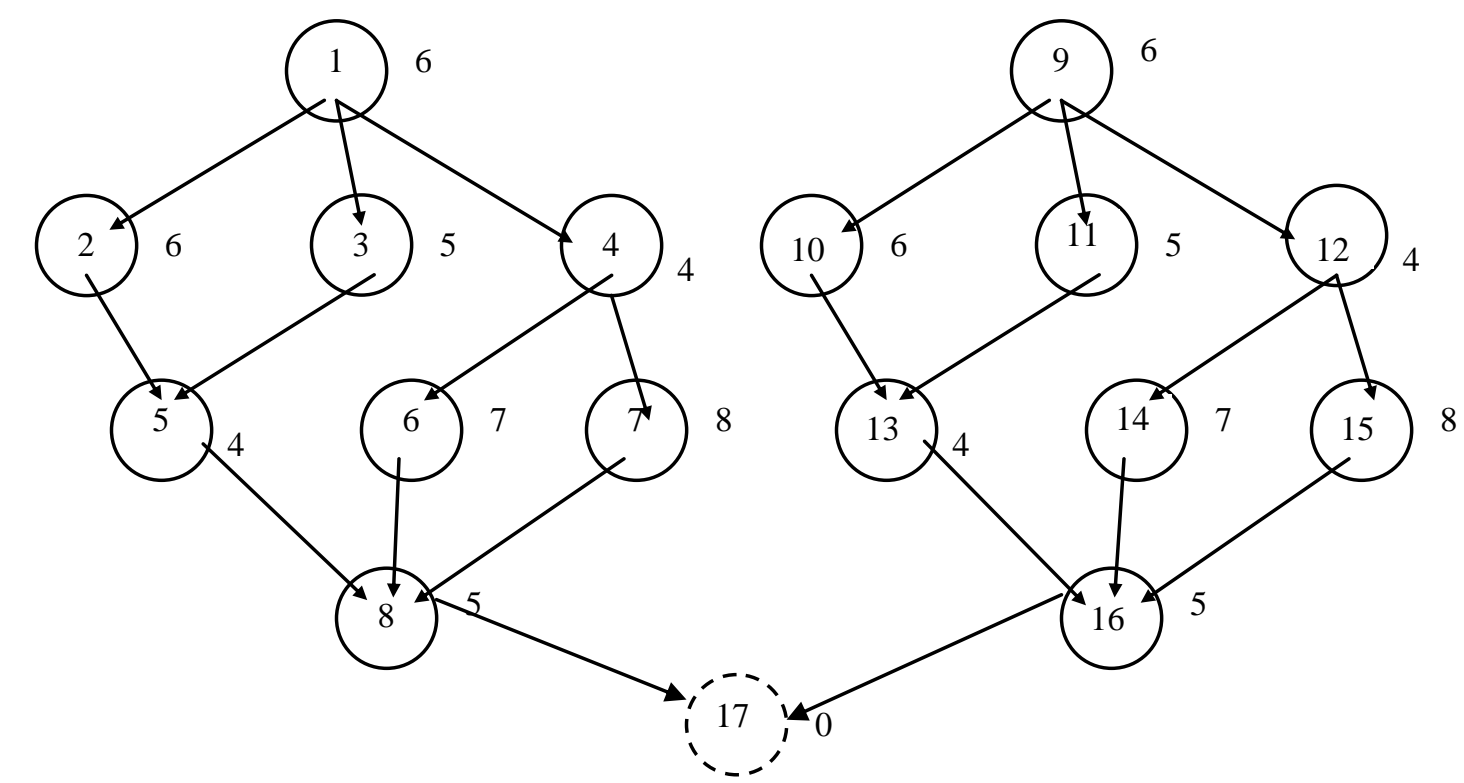

(Dash nodes representing dummy tasks)

Since most audit engagements are timing-crucial, a CPA firm can determine the minimum number of auditors needed to meet the required deadlines by implementing the heuristic scheduling model. This objective can be achieved by adding auditors to the engagement(s) until we find the lowest possible completion time of the engagements. According to the heuristic model, when the completion time of the terminal task is equal to the level of the starting task, this completion time is the lowest. For example, in Case 2, the completion time of the last task is 29 and the level of the first task is 23 . This means that adding more auditors to the engagement will decrease the completion time. On the other hand, in Case 3, one more auditor is added to the engagement and the completion time of the terminal task (i.e., 23) is equal to the level of the start task (i.e., 23) of the engagement. Therefore, 23, which uses three auditors, is the lowest completion time for this engagement, and assigning additional auditors to the engagement will not decrease the total completion time. Therefore, an optimal schedule has been derived in this situation.

\section{A Pair-Wise Exchange Model}

Pair-wise exchange concept has been used in the placement systems of very large-scale integrated circuits (Hanan, Wolff, and Agule 1976; Iosupovici, King, and Breuer 1983; Khokhani and Patel 1977). The performance of the heuristic model can be improved by interactively exchanging the order of tasks in the priority list while satisfying the precedence constraints. Let [i] represent the task with the $i^{\text {th }}$ priority, while $\alpha_{i}$ is the set of successors of task $i$ and $\beta_{\mathrm{i}}$ is the set of all parents of task $i$. The following lemma defines a feasible exchange of tasks in the priority list.

\section{Lemma:}

If $[1][2] \ldots[\mathrm{j}] \ldots[\mathrm{k}] \ldots[\mathrm{N}]$ is the original priority list, then $[1][2] \ldots[\mathrm{k}] . .[\mathrm{j}] \ldots[\mathrm{N}]$ is also a feasible priority list if and only if all the successors of task [j] are allocated after task [k] and all the parents of task $[\mathrm{k}]$ are executed before task [j], i.e., $\alpha[j] \subset\{[\mathrm{k}+1],[\mathrm{k}+2], \ldots[\mathrm{N}]\}$ and $\beta[\mathrm{k}] \subset\{[1],[2], \ldots,[\mathrm{j}-1]\}$. 
Proof: Necessary condition: trivial.

Sufficient condition:

It will be proven by contradiction. Since $[1],[2], \ldots[\mathrm{j}], \ldots[\mathrm{k}], \ldots[\mathrm{N}]$ is a feasible priority list, all the predecessors of task [j] must be executed before task [j], and all the successors of task [k] must be executed after task $[\mathrm{k}]$, i.e., $\beta_{[\mathrm{j}]} \subset\{[1],[2], \ldots,[\mathrm{j}-1]\}$ and $\alpha[\mathrm{k}] \subset\{[\mathrm{k}+1][\mathrm{k}+2], \ldots[\mathrm{N}]\}$. If any successor of task $[\mathrm{j}]$ or any predecessor of task $[\mathrm{k}]$ is executed between tasks $[\mathrm{j}]$ and $[\mathrm{k}]$, then an exchange on the order of execution of tasks [j] and [k] will require the execution of at least one task prior to its predecessor. That is, the new priority list will violate the precedence constraint. The proof is thus complete.

The starting and terminal tasks (i.e., tasks [1] and [N]) cannot be exchanged with any other tasks. The pairwise exchange model proceeds as follows. Given a directed task activity network graph $G_{t}=\left(V_{t}, E_{t}\right)$ with parameters $\left(s_{i}, d_{i}, a_{i}\right)$ and auditors with parameters $\left(\mu_{p}, e_{\mathrm{p}}, R_{p}\right)$, the pair-wise exchange model computes the assignments of tasks $\left(T_{1}, T_{2}, . ., T_{p}, . ., T_{M}\right)$, where $T_{p}$ is the ordered set of tasks assigned to auditor $p$.

Steps 1-3: Same as Steps 1-3 of the heuristic model.

Step 4: $\quad$ Improve the results systematically.

$$
\begin{aligned}
& \text { For } \mathrm{j}=2 \text { to } \mathrm{N}-2 \text { do } \\
& \text { flag }=0 \text {; } \\
& \text { For } \mathrm{k}=\mathrm{j}+1 \text { to } \mathrm{N}-1 \text { do } \\
& \text { If tasks [j] and }[\mathrm{k}] \text { are exchangeable, then } \\
& \text { Swap tasks }[\mathrm{j}] \text { and }[\mathrm{k}] \text { to construct a new priority list } \\
& \text { Use Step } 3 \text { of the heuristic algorithm to find a new scheduling } \\
& \text { If the result is better, then } \\
& \mathrm{k} \text { ' }=\mathrm{k} \\
& \text { flag }=1 \\
& \text { Update the result } \\
& \text { end if } \\
& \text { Swap tasks }[\mathrm{j}] \text { and }[\mathrm{k}] \\
& \text { end if } \\
& \text { end do } \\
& \text { If (flag equals } 1) \text { then } \\
& \text { Swap tasks [j] and }\left[\mathrm{k}^{\prime}\right] \\
& \text { end if } \\
& \text { end do }
\end{aligned}
$$$$
\text { Swap tasks [j] and }[\mathrm{k}] \text { to construct a new priority list }
$$

In Step 4, the inner do loop finds the best task [ $\left.\mathrm{k}^{\prime}\right]$ for the $\mathrm{j}^{\text {th }}$ position of the allocation order and the outer do loop controls the value of $\mathrm{j}$ and then exchanges the $\mathrm{j}^{\text {th }}$ position with the best task found, if it results in a shorter completion time. Since there exists a starting task and a terminal task in the acyclic graph, it is obvious that [ $\left.1^{\prime}\right]=1$ and $\left[\mathrm{N}^{\prime}\right]=\mathrm{N}$. Therefore, there are exchangeable tasks for both starting and terminal tasks in the activity network.

\section{Pair-wise Exchange Model with Different Levels of Efficiency and the Same Level of Expertise}

The pair-wise exchange model can be applied to Case 4. First, the heuristic scheduling model is applied to the allocation sequence 1, 4, 2, 3, 7, 6, 5, 8 to obtain an upper bound of the completion time, 19.33, for the engagement in Case 4 (Table 3). Then, the allocation sequence is modified by swapping [2] $=4,[3]=2$ to $[2]=2,[3]=4$, obtaining a new sequence $1,2,4,3,7,6,5,8$. Since all the successors of task $4,\left\{T_{6}, T_{7}\right\}$, are assigned after task 2 and all the parents of task $2,\left\{T_{1}\right\}$, are assigned before task 4 , these two tasks can be swapped. The heuristic scheduling model using the new allocation sequence produces a shorter completion time, 18.33. Therefore, [2] is assigned to audit task 2 , although the new sequence does not comply with the non-increasing levels. The final simulation results are shown in Table 6. The completion time of this engagement using pair-wise exchange algorithm is 
18.33. When compared to the completion time of 19.33 in Case 4 (see Table 3), the results of the pair-wise exchange algorithm result in a better audit schedule by one time unit (reduced from 19.33 time units to 18.33 time units). An exhaustive search has been applied to this case, and the results show that the pair-wise model does provide the optimal solution to this particular scheduling case.

\section{Pair-wise Exchange Model with Different Levels of Efficiency and Expertise}

The pair-wise exchange model can be applied to Case 5 as well. First, the heuristic scheduling model is applied to the allocation sequence $1,4,2,3,7,6,5,8$ to obtain an upper bound of the completion time, 26.33, for the engagement. Then, the allocation sequence is changed by swapping $[2]=4,[3]=2$ to $[2]=2,[3]=4$, obtaining a new sequence $1,2,4,3,7,6,5,8$. Since all the successors of task $4,\left\{T_{6}, T_{7}\right\}$, are assigned after task 2 and all the parents of task $2,\left\{T_{1}\right\}$, are assigned before task 4 , these two tasks can be swapped. The heuristic scheduling model using the new allocation sequence produces a shorter completion time, 22.33. Therefore, [2] is assigned to audit task 2 , although the new sequence does not comply with the non-increasing levels. The final simulation results are shown in Table 7. The completion time of this engagement using pair-wise exchange algorithm is 22.33 (see Table 7). When compared to the completion time of 26.33 using heuristic approach in Case 5, the results of the pair-wise exchange algorithm result in a better audit schedule by four time units (reduced from 26.33 time units to 22.33 time units). An exhaustive search has been applied to this case, and the results show that the pair-wise model does provide the optimal solution to this specific scheduling case.

\section{Pair-wise Exchange Model with Multiple Engagements}

Similar to Cases 4 and 5, the pair-wise exchange model can be applied to multiple engagements. In Case 6, the model swapped the sequence of audit tasks 4 and 12. The final simulation results are shown in Table 8 . The completion time of this engagement using pair-wise exchange algorithm is 40.00 , compared to the completion time of 42.33 in Table 5; that is, the results of the pair-wise exchange algorithm result in a better audit schedule by 2.33 time. An exhaustive search has been applied to this case, and the results show that the pair-wise model does provide the optimal solution to this scheduling case.

\section{Summary and Conclusion}

During the past decade, the business environment of the public accounting has become very competitive. Changes in the operating environment have posed a number of challenges for accounting firms' top executives. One of the critical issues faced by accounting firm management is how to minimize their audit costs while maintaining high audit quality. As proposed by many, one possible approach to fulfilling this objective is to employ a systematic and easy-to-use scheduling approach. In this study, I first propose a heuristic scheduling approach and then to improve its scheduling results by using pair-wise exchange model. The pair-wise exchange model considers minimizing the engagement completion time subject to the available human resource in a given planning horizon, the different levels of auditors' expertise required, precedence constraints of the audit tasks, and the due dates of the engagement.

There are some merits to the proposed method. It is easy to implement and particularly suitable for large size public accounting practices. The level from critical path method is used as the lower bound of audit staff scheduling, and the level's non-increasing concept is applied to prioritize the human resource planning in an accounting firm. Based on the priority list, a heuristic model is developed and a pair-wise exchange model is then implemented to seek additional time saving. The heuristic model is an efficient means for creating a desirable schedule for an audit engagement. Results of the proposed heuristic model are used as the starting point for developing the pair-wise exchange model in this study. By swapping the priority of task sequences, the pair-wise exchange algorithm can be applied to systematically improve the overall schedule. Furthermore, constraints can easily be added to or removed from these two models by performing or deleting extra checks from the assigning steps of the heuristic model. The model proposed in this paper would move us toward a better solution to a common concern in public accounting practice. 


\section{Suggestions for Future Research}

The nature of staff scheduling is very complicated and poses multi-dimensional problems (Chan, Lam and Cheng 1998; Cai and Li 2000; Dean et al. 1992; Dumond and Marbert 1988; Hwang 1998; and Kurtalus and Davis 1982). This study develops a scheduling approach that is useful in a competitive public accounting environment. Future studies should test this proposed scheduling approach in a real-world environment. Upon completion of testing, it will be possible to compare the efficiency and practicality of this model to other models currently being used for audit planning. It is also important to note that there is no easy or perfect solution to the problems of scheduling audit staff. Even so, accounting researchers may explore dynamic approaches to staff scheduling and/or human resource allocation for CPA firms. A dynamic model should be effective to the public accounting firms in human resource management. 미

\section{References}

1. Balachandran, B. V. and A. A. Zoltners, "An Interactive Audit-Staff Scheduling Decision Support System," The Accounting Review, Vol. 56, No. 4, pp. 801-812, 1981.

2. Balanchandran, K. R. and R. E. Steuer, "An Interactive Model for the CPA Firm Audit Staff Planning Problem with Multiple Objectives," The Accounting Review, Vol. 57, No. 1, pp. 125-139, 1982.

3. Chan, K. H. and B. Dodin, "A Decision Support System for Audit Staff Scheduling with Precedence Constraints and Due Dates," The Accounting Review, Vol. 59, No. 4, pp. 726-734, 1986.

4. _ S. F. Lam, and T. T. Cheng, "Audit Scheduling with Limited Resources," CA Magazine (November), pp. 38-44, 1978.

5. _ , and S. Cheng, "Audit Scheduling and the Control of Travel Costs Using an Optimization Model for Multinational and Multi-location Audits," Journal of Accounting, Auditing and Finance, Vol. 13, No. 1, pp. 67-98, 1998.

6. Cai, X., and K. N. Li, “A Genetic Algorithm for Scheduling Staff of Mixed Skills under Multi-criteria," European Journal of Operational Research, Vol. 125, No. 2, pp. 359-369, 2000.

7. Dean, B. V., D. R. Denzler, and J.J. Watkins, "Multiple Staff Scheduling Methods with Variable Resource Constraints," IEEE Transactions on Engineering Management (February), pp. 59-72, 1992.

8. Dodin, B. and K. H. Chan, "Application of Production Scheduling Methods to External and Internal Audit Scheduling," European Journal of Operational Research, Vol. 52, pp. 267-279, 1991.

9. Dumond, J., and V. A. Mabert, "Evaluating Project Scheduling and Due Date Assignment Procedures: An Experimental Analysis," Management Sciences, Vol. 34, pp. 101-118, 1988.

10. Gardner, J. C., R. J. Huefner, and V. Lotfi, "A Multiperiod Audit Staff Planning Model Using Multiple Objectives: Development and Evaluation,” Decision Sciences, Vol. 21, pp. 154-170, 1990.

11. Hamburger, D., "On-time Project Completion - Managing the Critical Path," Project Management Journal, Vol. 18, No. 4, pp. 79-85, 1987.

12. Hanan, M., P. K. Wolff, and B. J. Agule, "Some Experimental Results on Placement Techniques," Proceedings of the $13^{\text {th }}$ Design Automation Conference, Vol. 214-224, 1976.

13. Hwang, N. C., "An Auditor Scheduling Approach in a Competitive CPA Environment," Asia-Pacific Journal of Accounting, Vol. 5, No. 1, pp. 25-44, 1998.

14. Iosupovici, A., C. King, and M. A. Breuer, “A Module Interchange Placement Machine," Proceedings of the $20^{\text {th }}$ Design Automation Conference, pp. 171-174, 1983.

15. Kasahara, H. and S. Narita, "Practical Multiprocessor Scheduling Algorithms for Efficient Parallel Processing," IEEE Transactions on Computers C-33, Vol. 11, pp. 1023-1029, 1984.

16. Khokhani, K. H. and A. M. Patel, "The Chip Layout Problem: A Placement Procedure for LSI," Proceedings of the $14^{\text {th }}$ Design Automation Conference, PP. 291-297, 1977.

17. Krogstad, J., L. Grudniski, and D. Bryant, "PERT and PERT/Cost for Audit Planning and Control," Journal of Accountancy (November), pp. 82-91, 1977.

18. Kurtalus, I. and E. W. Davis, "Multi-project Scheduling: Categorization of Heuristic Rule Performance," Management Science, Vol. 28, No. 2, pp. 161-172, 1982.

19. Summers, E. L., "The Audit Staff Assignment Problem: A Linear Programming Analysis," The Accounting Review, Vol. 47, No. 3, pp. 443-453, 1972. 
20. Thompson, G. M., "Controlling Action Times in Daily Workforce Schedules," Cornell Hotel and Restaurant Administration Quarterly (April), pp. 82-96, 1996.

21. Ying, C.C., "Productivity and Staffing Analysis of a Sterile Processing Department," Computers and Industrial Engineering (October), pp. 459-461, 1996.

\section{Endnotes}

i The objectives can be categorized as (1) satisfaction of the generally accepted auditing standards while minimizing the amount of time spent in an engagement, (2) maximization of profit, (3) balancing the work load among auditors, (4) minimization of overtime, (5) full utilization of workforce, (6) minimization of the time allocated to an audit engagement, and (7) curtailment of total delay cost.

ii An audit task can be processed by different auditors with different levels of efficiency. Therefore, the duration time of each task depends upon who processes it.

iii Auditors at different levels perform different tasks. Mismatches can cause higher costs (e.g., assigning a manager to perform a senior's task) or an ineffective audit (e.g., assigning a junior audit staff to perform a senior's task).

iv Chan and Dodin (1986) argue that for large and multiple engagements to be scheduled simultaneously and when audit tasks are interrelated, it is not feasible to find out beforehand what tasks will run concurrently. Therefore, it is also a constraint of the proposed model in this study.

$v \quad$ If needed, the objective function can be modified to solve the mismatches or total penalty of lateness.

vi In this study, I consider only auditors' salary as an audit cost and ignore other supporting expenditures.

vii The simulation program is written in $\mathrm{C}++$. 